\title{
Review
}

\section{Degradation of Misfolded Proteins by Autophagy: Is it a Strategy for Huntington's Disease Treatment?}

\author{
Fang Lin and Zheng-Hong Qin* \\ Department of Pharmacology, Laboratory of Aging and Nervous Diseases, Soochow University School \\ of Pharmaceutical Science, Suzhou, China
}

\begin{abstract}
Autophagy is a degradation pathway for long-lived cytoplasmic proteins, protein complexes, or damaged organelles. The accumulation and aggregation of misfolded proteins are hallmarks of several neurodegenerative diseases. Many researchers have reported that autophagy degrades disease-causing misfolded and aggregated proteins, including mutant huntingtin (Htt) in Huntington's disease, mutant synuclein in familial Parkingson's disease, mutant $\mathrm{Cu}, \mathrm{Zn}^{-}$Superoxide dismutase (SOD1) in familial amyotrophic lateral sclerosis. In this review, we will bring up new evidence to elucidate the involvement of autophagy in degradation of mutant Htt, discuss the mechanisms regulating the degradation of mutant Htt by autophagy and the therapeutic effects of drugs that enhance autophagy to improve clearance of mutant $\mathrm{Htt}$. We propose that enhancement of autophagy by drugs may be a strategy to treat or retard progression of Huntington's disease.
\end{abstract}

Keywords: Huntington's disease, huntingtin, polyglutamine expansion, autophagy

\section{MUTANT HUNTINGTIN AGGREGATES IS A HALLMARK OF HUNTINGTON'S DISEASE}

Huntington's disease (HD) is an inherited neurodegenerative disease which is characterized by the accumulation of mutant huntingtin $(\mathrm{Htt})$ protein that contains abnormal polyglutamine (polyQ) expansion encoded by expanded CAG $[1,2]$. Htt protein can be cleaved by caspases, calpains and aspartic proteases to form short $\mathrm{N}$-terminal fragments with the expanded polyQ [3]. Mutant $\mathrm{Htt}$ with greater than 37 polyQ undergoes conformational changes from alpha-helix

*Correspondence to: Zheng-Hong Qin, Soochow University School of Pharmaceutical Science, 199 Ren Ai Road, Suzhou, Post code: 215 123, China. Tel.: +86 512 65882071; E-mail: zhqin5@hotmail.com. to beta-sheet to form aggregates in vitro or in brains of animal models and HD patients [4]. The shorter Nterminal or longer CAG stretches tend to form more aggregates [5]. Additionally, the flanking sequences surrounding the polyQ region can also play a critical role in determining the structural rearrangement and aggregation [6]. Mutant Htt aggregates are found in the nucleus, the cytoplasm and in processes and, are a pathological hallmark in HD [7]. Mutant Htt acquires abnormal protein interactions and sequestration with other normal cellular proteins, such as heat shock proteins, some transcriptional factors and proteins related to vesicle trafficking, causing loss of normal physiological functions of $\mathrm{Htt}$ and gain of toxic functions. Several studies reported a strong correlation between the accumulation of the mutant protein and disease severity $[8,9]$. 


\section{FEATURES OF PROTEIN DEGRADATION BY AUTOPHAGY VS UPS}

Proteins can be degraded by the ubiquitinproteasome system (UPS) and the autophagylysosome pathway. The UPS pathway consists of ubiquitination of substrates and proteasome-mediated degradation. The substrates are delivered for degradation after they are tagged with polyubiquitin chains. UPS predominantly degrades short-lived normal proteins, e.g., proteins involved in regulation of cell division, gene transcription, signal transduction, and endocytosis, after they have fulfilled their duty in the cells [10-12]. UPS is also a critical step of posttranslational protein quality control (PQC) in the cells. Abnormal proteins, such as misfolded, oxidized, or mutant proteins will be ubiquitinated and degraded in the proteasome [13]. However, the narrow pore of the proteasome precludes entry of protein complexes and organelles. Hence, the bulk degradation of cytoplasmic proteins or organelles is mediated largely by autophagy.

Autophagy (self-eating) refers to any intracellular processes that result in the degradation of cytosolic components inside lysosomes. Based on the ways by which substrates reach the lysosomal lumen, autophagy is divided into three major forms: macroautophagy, microautophagy and chaperone mediated autophagy (CMA). Autophagy plays a role in cell homeostasis, cell differentiation, tissue remodeling, growth control, starvation response, innate and adaptive immunodefense, and adaptation to adverse environments [14, 15].

Macroautophagy is characterized by formation of double-membrane vesicles around portions of cytoplasm to form autophagosomes which eventually fuse with lysosomes, where their contents are degraded. The substrates of macroautophagy include cytoplasmic proteins, misfolded proteins, mitochondria, peroxisomes and regions of Golgi and endoplasmic reticulum. Although there is evidence showing that macroautophagy has selectivity towards its substrates [16-18], the nature of autophagosome formation suggests low selective degradation mediated by macroautophagy.

Compared with macroautophagy, CMA has higher selectivity. The substrates of CMA containing KFERQ motifs can be recognized by heat shock cognate protein of $70 \mathrm{kDa}$ (Hsc70), thus facilitating the delivery of the proteins to lysosomes through Lysosome-associated membrane protein 2 (LAMP-2A) [19-21]. LAMP$2 \mathrm{~A}$ functions as a receptor and makes pores for the uptake of substrates into lysosomes, while Hsc70 is the only chaperone demonstrated to mediate substrate targeting for CMA. The levels of Hsc70 and LAMP-2A determine the activity of CMA. Unlike macroautophagy which is a conserved physiological phenomenon, CMA has only been described in mammals.

Both the proteasome system and autophagy are critical protein degradation pathways [22]. Autophagy may be upregulated by an impaired proteasome pathway. Blockage of macroautophagy and proteasome function would result in constitutive activation of CMA.

$\mathrm{Htt}$ has been suggested to be ubiquitinated for UPS degradation [23]. Hence, proteasome pathway may play an important role in degrading the oligomerized mutant Htt in the cytoplasm and the nucleus [24-26]. However, it has been proposed that a hairpin loop created by the expanded polyQ stretch-forms a highly stable $\beta$-sheet structure that inhibits unfolding and degradation by the proteasome [27]. It was also reported that ubiquitinated mutant Htt accumulates in cells $[28,29]$. Hence, autophagy could play an important role in the degradation of mutant Htt, esp. aggregate degradation (Fig. 1).

\section{AUTOPHAGY PLAYS AN IMPORTANT ROLE IN MUTANT HTT CLEARANCE}

\section{Autophagy is altered in $H D$}

A number of studies have reported that autophagy is increased in HD. DiFiglia's laboratory first reported that $\mathrm{Htt}$ markedly accumulated in endosomal-lysosomal organelles of affected HD neurons, and Htt-labeled vacuoles displayed ultrastructural features of early and late autophagosomes in HD patients and in HD cell models [30-32]. Some research showed that macroautophagy appeared to be disrupted by mutant Htt, that autophagic vacuoles formed at normal or even enhanced rates in HD, mutant $\mathrm{Htt}$ interacted with vesicles and interfered with the recognition of cytosolic cargo, compromising the intracellular turnover of cytosolic organelles [33].

The decline in levels of macroautophagy with age $[34,35]$ may partly account for the pathogenesis of HD and other diseases with mutant protein aggregates that strike individuals mainly in midlife. Interestingly, expression of full-length Htt lacking its polyglutamine stretch in HD knock-in mice enhanced autophagy, and mice showed reduced mutant $\mathrm{Htt}$ aggregates with amelioration of motor/behavioral deficits, and extended lifespan. These results suggest that increased 


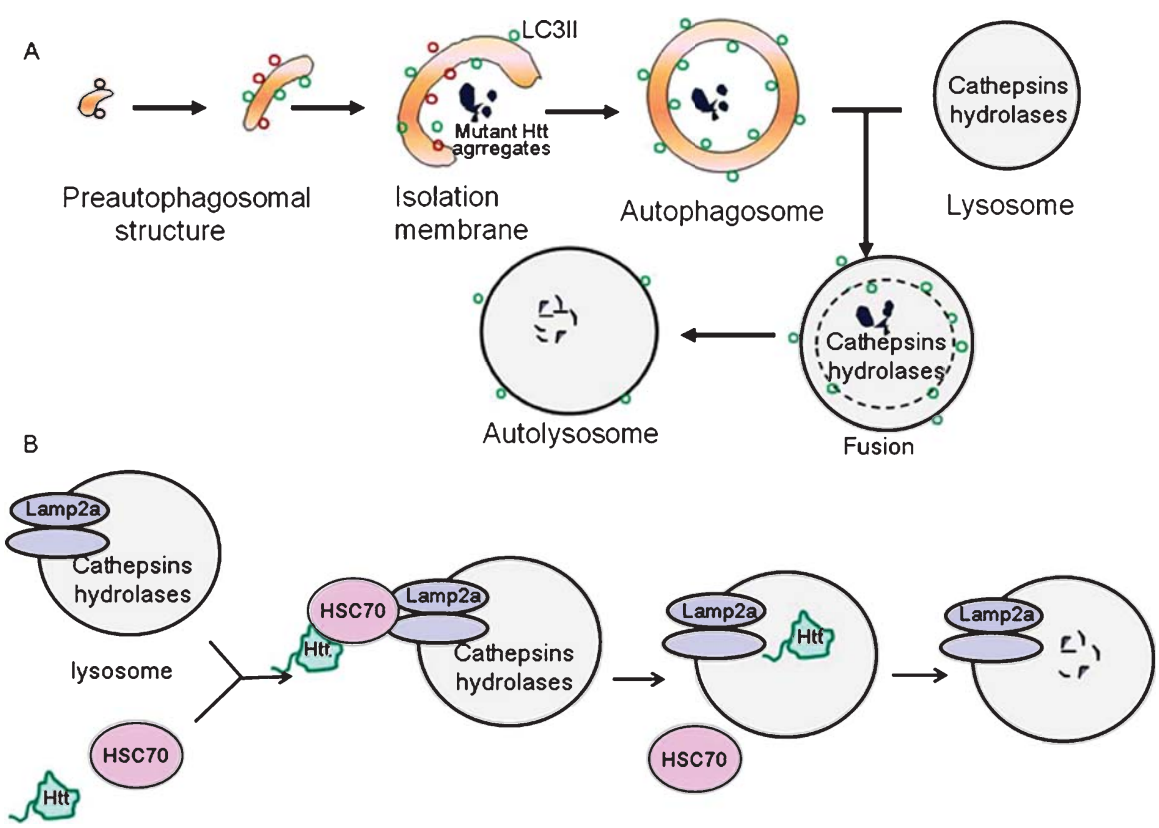

Fig. 1. A. The degradation of $\mathrm{Htt}$ and mutant Httt aggregates by macroautophagy. B. The degradation of wild and mutant Htt by chaperon mediated autophagy.

autophagy may alleviate HD onset and promote lifespan in normal mammals [36]. Furthermore, a genetic study of 900 European HD patients showed that the presence of a substitution of alanine for valine (V471A) in the autophagy gene atg7 was associated with an earlier disease onset of 4 years in some HD populations [37].

\section{Htt can be degraded by macroautophagy}

Qin et al. reported that Htt expression increased levels of the lysosomal enzyme cathepsin D by an autophagy-dependent pathway in mouse clonal striatal cells x57 expressing truncated and full-length human wild-type and mutant Htt [2]. Cells without cathepsin $\mathrm{D}$ accumulated more $\mathrm{N}$-terminal $\mathrm{Htt}$ fragments, suggesting that autophagy plays a critical role in the degradation of N-terminal Htt. Several other studies provided support that macroautophagy contributes to the clearance of mutant $\mathrm{Htt}$ and that pharmacological upregulation of autophagy alleviates disease-related symptoms in animal models [38-41]. The autophagosome formation inhibitor 3MA or the autophagolysosome formation inhibitor Bafilomycin A1 increased Htt aggregates in cells while the autophagy inducer rapamycin reduced Htt aggregates. The research also showed that the behavioral performance of HD mice improved with decreased
Htt aggregation due to the autophagy inducer. These results suggest that autophagy plays a potential role in enhancing the turnover of mutant $\mathrm{Htt}$, and this process could alleviate HD symptoms.

Several scaffold proteins affect mutant Htt aggregate degradation. Insulin receptor substrate 2 (IRS-2), which mediates the signaling cascades, leads to a macroautophagy-mediated clearance of mutant Htt [42]. Alfy, interacting with p62 and NBR1, is essential for the macroautophagic clearance of ubiquitin-positive inclusions [43]. Alfy overexpression decreased mutant $\mathrm{Htt}$ aggregates and protected cells from the toxicity of mutant $\mathrm{Htt}$ in a primary neuronal model and a fly model. Additionally, transcription factor PPAR $\gamma$ co-activator $1 \alpha$ (PGC-1 $\alpha)$ eliminated protein aggregates by activating transcription factor EB (TFEB), which is a key regulator of the autophagy-lysosome pathway. PGC- $1 \alpha$ rescued TFEB level repressed by mutant $\mathrm{Htt}$ and enhanced autophagylysosome pathway activity [44].

Some studies have demonstrated that the selective degeneration of striatal neurons was partly due to dysfunctional glia in HD pathology [45, 46]. GLT-1 is a glutamate transporter which can clear extracellular excitatory neurotransmitters to protect neurons from excitatory amino acid toxicity. Chen et al. found that the GLT-1 level as well as the uptake capability of glutamate decreased in astrocytes expressing mutant $\mathrm{Htt}$ 
[47]. Rapamycin treatment upregulated autophagy in glia expressing mutant $\mathrm{Htt}$, and recovered the level of GLT-1 and the uptake capability of glutamate.

\section{Htt can be degraded by CMA}

Both Hsc70 and LAMP-2A are vital proteins for CMA degradation. Experimental upregulation of CMA by overexpressing LAMP-2A reduces intracellular protein inclusions and increases cellular viability in cultured neuronal cells expressing posttranslationally modified forms of mutant $\mathrm{Htt}$ [48]. In addition, a 46 amino acid-peptide adaptor molecule comprising two copies of the polyglutamine-binding peptide and two different Hsc70-binding motifs was shown to direct mutant Htt to the CMA machinery for degradation. Viral delivery of this construct to the striatum in the R6/2 mouse model of HD significantly reduced polyQ aggregation in the transduced areas, ameliorated symptoms and extended lifespan [49]. Koga et al. found that the full-length wild-type or mutant protein was not normally degraded via CMA although there may be three KFERQ-like motifs in the Htt sequence, whereas the N-terminal of both wild-type and mutant Htt are susceptible to CMA [50]. Recently, Qi et al. reported that ${ }_{99} \mathrm{KDRVN}_{103}$ of $\mathrm{Htt}-552$ is a functional site for substrate recognition by CMA [51]. In HD, CMA is upregulated constitutively, but the decline with age in efficiency of CMA-mediated degradation may be associated with cellular failure and onset of pathological manifestations $[52,53]$.

\section{Post-translational modifications of Htt enhance autophagy-mediated degradation}

Post-translational modification (PTM) is chemical modification on proteins after translation. Different post-translational modifications of $\mathrm{Htt}$, such as SUMOylation, ubiquitination, phosphorylation, palmitoylation and acetylation, will play diverse roles in cellular function. In this review, we just focus on phosphorylation and acetylation, which were reported to be associated with Htt degradation by autophagy.

Phosphorylation regulates protein degradation, and alters subcellular localization and secondary modifications such as ubiquitination, SUMOylation and acetylation. Gu et al. reported that changes in the phosphorylation at serines 13 and 16 of Htt would impact the aggregation properties of Htt [54]. They found that disease pathogenesis induced by mutant $\mathrm{Htt}$, including motor and psychiatric-like behavioral deficits, mutant Htt aggregation and selective neurodegeneration were abolished by expressing full-length mutant huntingtin with serines 13 and 16 mutated to aspartate (phosphomimetic). Thompson et al. found that $\mathrm{Htt}$ was phosphorylated at $\mathrm{S} 13$ by the inflammatory kinase IKK directly [48]. Pharmacological activation of IKK with IL-1 $\beta$ or TNF- $\alpha$ enhanced the phosphorylation of $\mathrm{Htt}$ S13. IKK- $\beta$ increased phosphorylation and reduced levels of normal polyQ Htt fragments in a manner dependent on both the proteasome and lysosome, while expanded the polyQ repeat inhibited this effect. Furthermore, knockdown of either endogenous LAMP-2A or Atg7 by RNA interference increased the accumulation of endogenous S13-phosphorylated $\mathrm{Htt}$ and $\mathrm{Htt}$ fragments, suggesting that both CMA and macroautophagy are involved in phosphorylated wild-type $\mathrm{Htt}$ clearance.

Steffan et al. demonstrated that mutant Htt interacts directly with the histone acetyltransferase (HAT) domain of CREB binding protein (CBP) [55]. Later, Jeong et al. identified an acetylated lysine at position 444 (K444) in human Htt [56]. Acetylated mutant Htt was trafficked to autophagosomes for degradation, while the non-acetylated mutant $\mathrm{Htt}$ was prone to form aggregates. The de-acetylation process is catalyzed by histone deacetylase 1 (HDAC1). The pan-HDAC inhibitor SAHA (Suberoylanilide hydroxamic acid) has been shown to improve phenotypes in the R6/2 mouse model, providing evidence for the therapeutic usefulness of this class of compounds [57]. Interestingly, some recent studies showed that SAHA could induce autophagy $[58,59]$. It would be interesting to further confirm whether the effect of SAHA on pathological improvement in HD animal models is associated with mutant Htt degradation by autophagy enhancement. HDACi 4 b, which preferentially targets HDAC1 and HDAC3, could ameliorate the phenotypes in HD transgenic mice [60]. Jia et al. recently reported that HDACi $4 \mathrm{~b}$ prevented the formation of nuclear Htt aggregates in the brains of N171-82Q mice through modulation of the ubiquitin-proteasomal and autophagy pathways [61].

\section{Some issues in the study of mutant Htt clearance}

Although there has been progress in understanding pathways of mutant htt clearance, several technical issues need to be further considered. For example, how to detect the efficiency of Htt clearance? Generally, Western blot or immunohistochemistry methods are utilized to detect Htt levels. However, Htt is a protein with more than 3000 amino acids. One cannot conclude that Htt clearance has occurred based on the 
loss of signal with one antibody. Different antibodies identifying different epitopes in Htt should be utilized in the same experiment to confirm the levels of Htt. Another important issue of concern is the HD models to be used. Generally, N-terminal fragments of $\mathrm{Htt}$ have been used to study autophagy in cells since it is difficult to manipulate the expression of the full length Htt gene. Although N-terminal fragments of mutant Htt expressed in mice produce a HD-like pathology, the metabolism and role of these $\mathrm{N}$-terminal fragments may be different from full-length Htt. Many previous studies have used various cell lines for studies of autophagy. Researchers should focus more on neurons and other cells in central nervous system since the main pathology of HD is in striatum and cortex. However, it is difficult to express exogenous genes in neurons. As such, research with induced pluripotent stem (IPS) from HD patients may be a good approach.

\section{POTENTIAL THERAPEUTIC STRATEGY FOR HD BY AUTOPHAGY ACTIVATION}

Currently, tetrabenazine is the only FDA approved drug for HD treatment to control movement disorder [62-64].

Since HD is caused by neuronal accumulation of mutant Htt, improving clearance of the mutant protein is expected to prevent cellular dysfunction and neurodegeneration in HD. Several groups have been working to develop autophagy upregulating drugs for the purpose of treating aggregate-prone neurodegeneration. In Rubinsztein's laboratory, a high-throughput screen identified various small molecule inhibitors (SMIRs) and enhancers (SMERs) of the growthsuppressing properties of rapamycin in yeast. Three SMERs (SMERs 10, 18 and 28) were subsequently confirmed to induce mTOR-independent autophagy in mammalian cells and they increased clearance of mutant Htt and reduced Htt toxicity in the Drosophila HD model [65]. In another drug screening, several FDA (US Food and Drug Administration)-approved drugs/compounds (e.g., verapamil, loperamide amiodarone and clonidine) were found to induce autophagy and decrease mutant Htt aggregates and confer protection against toxicity in HD cellular, fly and zebrafish models [66]. Using an automated cell-based assay, Roberge's laboratory screened a collection of 3,500 chemicals and identified three approved drugs (perhexiline, niclosamide, amiodarone) and one pharmacological reagent (rottlerin) capable of rapidly increasing autophagosome contents [67]. In a neuron model of HD, Tsvetkov et al. found that 10[4'-(N-diethylamino)butyl]-2-chlorophenoxazine (10NCP), an N10-substituted phenoxazine, up-regulated autophagy in an mTOR-independent fashion in primary neurons, decreased the accumulation of diffuse and aggregated mutant Htt and exerted a neuroprotective effect [68]. Furthermore, the authors screened other structural analogues of 10-NCP that had been FDA approved. Some compounds in different classes of the tricyclics, including trifluoperazine, promazine and its analogs, promethazine, thioridazine, and mesoridazine were verified to trigger autophagy in neurons. However, most of these drugs have side effects on the extrapyramidal system, potentially worsening the movement disorder of HD.

\section{Rapamycin and temsirolimus}

Rapamycin is a lipophilic macrolide antibiotic originally used as an immunosuppressant. In mammalian cells, rapamycin inhibits the kinase activity of mTOR that suppresses autophagy. Rapamycin upregulates autophagy, enhances the clearance of mutant Htt fragments, thus reduces aggregate formation and protects against toxicity in cell, Drosophila and mouse models of HD. The water-soluble ester of the rapamycin analog temsirolimus (CCI-779) also reduces mutant $\mathrm{Htt}$ levels, and attenuates toxicity in mouse models of HD [69]. Similarly, rapamycin and its analog alleviate aggregation and symptoms in spinocerebellar ataxia type 3 models [70]. Since rapamycin was first reported to clear mutant Htt by the autophagy pathway [71], it was studied for therapeutic effects in a wide range of aggregate-prone proteins with polyglutamine- or polyalanine expansion. Although rapamycin as a drug could be used long term, it still has side effects due to inhibition of mTOR, which regulates many other cellular processes independent of autophagy. Therefore it is a priority to identify other drugs that induce autophagy via mTOR-independent mechanisms.

\section{Trehalose}

Trehalose, a disaccharide, is a naturally occurring reducer of cell stress, protecting these organisms from extremes in heat shock and osmotic stress [72]. Trehalose can maintain the three-dimensional structure of these biologic molecules under stress and preserve their biologic functions [73].

Tanaka et al. discovered trehalose in a screen which was designed to find any disaccharides that could inhibit polyglutamine mediated protein aggregation 
[74]. Oral administration of trehalose (2\% trehalose in the drinking water, spontaneous drinking) reduced the weight loss of R6/2 HD transgenic mice, whereas it did not affect the body weight of wild-type mice. Trehalose also ameliorated motor symptoms, decreased the number and size of aggregates, and prolonged life by $20 \%$ in the R6/2 transgenic mouse model of HD. Later, Sarkar et al. reported that trehalose induced autophagy in mTOR-independent manner in COS-7 (non-neuronal) and SK-N-SH (neuronal) cells, and reduced polyQmediated aggregation and cell death, and enhanced the clearance of soluble mutant Htt [39].

According to these studies, trehalose has potential to delay onset or decrease progression rate in HD and has become a supplement for HD patients. Trehalose is very safe and has been approved for use in the United States since it has protective effects in promoting brain health. Therefore, trehalose may have benefit for both prevention and treatment of neurodegenerative diseases.

\section{Rilmenidine}

Rilmenidine is a well-tolerated and centrally acting anti-hypertensive drug. Rose et al. reported that rilmenidine increased LC3-II significantly in stable inducible PC12 cells expressing either wild-type (23Q) or expanded polyglutamine (74Q) Htt [75]. Further research found that rilmenidine induced autophagy in mice and in primary neuronal culture. Rilmenidine had no effect on phosphorylation of p70 S6 kinase, S6 protein or 4E-BP1, and all downstream substrates of mTOR kinase activity, suggesting that, unlike the autophagy enhancing drug rapamycin, rilmenidine does not act to upregulate autophagy via inhibition of mTOR. Rilmenidine administration attenuated the signs of disease in a HD mouse model, including improvement on grip strength, tremors and wire traversing, which was comparable to the effect of rapamycin. In addition to the enhancement in movement, rilmenidine also reduced mutant $\mathrm{Htt}$ fragments. However, rilmenidine treatment did not protect against weight loss and shortened lifespan of the HD transgenic mice.

As an antihypertension drug, rilmenidine is more selective for imidazoline receptors than for $\alpha 2$ adrenoceptors and thus causes fewer central adverse effects such as sedation or antinociception. Because rilmenidine has a long safety record and is designed for chronic use, it is a strong candidate to consider for human trials in HD patients.

\section{Lithium, valproate and carbamazepine}

All three drugs are inositol-lowering drugs, which can induce autophagy by inhibiting inositol monophosphatase (IMPase), leading to depletion of intracellular inositol levels and inhibition of the phosphoinositol cycle. Indeed, these drugs enhance mutant Htt clearance and protect against its toxicity in cells and in vivo models $[66,76,77]$. Additionally, lithium, valproate and carbamazepine are in clinical use. Among them, lithium can be used to treat bipolar mood disorder, carbamazepine can be used to treat schizophrenia, and valproate can be used to treat epilepsy and schizophrenia. Hence, these compounds would be helpful to HD patients with depression or schizophrenia. However, lithium requires strict monitoring as it works within a relatively narrow therapeutic range.

Nevertheless, the above drugs need further clinical review to address their efficacy and safety for treating HD patients. Such efforts if successful could yield a drug therapy that both attenuates symptoms and slows disease progression in HD.

\section{CALORIC RESTRICTION, EXERCISE, AND AUTOPHAGY}

In addition to the drugs/reagents described above, caloric restriction and exercise also affect autophagy. Duan et al. reported that dietary restriction increased levels of brain-derived neurotrophic factor and heatshock protein-70 in the striatum and cortex, retarded the formation of Htt inclusions, apoptotic protease activation, enhanced movement in HD mice and extended lifespan [78]. Autophagy may be activated in caloric restriction because it is an adaptive response to nutrient starvation. Recently, some researchers showed that autophagy played a role in muscle establishment after exercise [79-82]. In our laboratory, we found that exercise ameliorated the detrimental effect of chloroquine on skeletal muscles by restoring autophagy flux (submitted). Muscle weakness is one of the symptoms of HD patients. Increased exercise could be helpful to upregulate autophagy in skeletal muscle and maintain muscle function and muscle mass in HD.

About two decades ago, the mutant gene causing HD was isolated and pathology and molecular pathological mechanisms were largely uncovered. In the last 10 years, many studies have revealed the physiological roles of autophagy in HD. In the next 10 years, we anticipate that the knowledge gained about autophagy and mutant Htt clearance could bring forth new treatments for the disease. 


\section{ACKNOWLEDGMENTS}

We are grateful to Dr. Shouqing Luo for his suggestions to this review. This work is supported by grants from the National Natural Science Foundation of China (No. 30600197), the National Basic Science Key Project (973 project CB51003), and the Priority Academic Program Development of Jiangsu Higher Education Institutes (PAPD).

\section{CONFLICT OF INTEREST}

The authors have no conflict of interest to report.

\section{REFERENCES}

[1] A novel gene containing a trinucleotide repeat that is expanded and unstable on Huntington's disease chromosomes. The Huntington's Disease Collaborative Research Group. Cell 1993;72:971-83.

[2] Qin ZH, Wang Y, Kegel KB, Kazantsev A, Apostol BL, Thompson LM, et al. Autophagy regulates the processing of amino terminal huntingtin fragments. Hum Mol Genet. 2003; $12: 3231-44$.

[3] Wellington CL, Singaraja R, Ellerby L, Savill J, Roy S, Leavitt $\mathrm{B}$, et al. Inhibiting caspase cleavage of huntingtin reduces toxicity and aggregate formation in neuronal and nonneuronal cells. J Bio Chem. 2000;275:19831-8.

[4] Perutz MF, Pope BJ, Owen D, Wanker EE, Scherzinger E. Aggregation of proteins with expanded glutamine and alanine repeats of the glutamine-rich and asparagine-rich domains of Sup35 and of the amyloid beta-peptide of amyloid plaques. Proc Natl Acad Sci USA. 2002;99:5596-600.

[5] Cooper JK, Schilling G, Peters MF, Herring WJ, Sharp AH, Kaminsky Z, et al. Truncated N-terminal fragments of huntingtin with expanded glutamine repeats form nuclear and cytoplasmic aggregates in cell culture. Hum Mol Genet. 1998;7:783-90.

[6] Lakhani VV, Ding F, Dokholyan NV. Polyglutamine induced misfolding of huntingtin exon1 is modulated by the flanking sequences. PLoS Comput Biol. 2010;6:e1000772.

[7] DiFiglia M, Sapp E, Chase KO, Davies SW, Bates GP, Vonsattel JP, et al. Aggregation of huntingtin in neuronal intranuclear inclusions and dystrophic neurites in brain. Science. 1997;277:1990-3.

[8] Rubinsztein DC, Huntington JA. Paradoxical aggregation versus oligomerisation properties of mutant and wild-type huntingtin fragments. Exp Neurol. 2006;199:243-4.

[9] Haass C, Selkoe DJ. Soluble protein oligomers in neurodegeneration: Lessons from the Alzheimer's amyloid beta-peptide. Nat Rev Mol Cell Biol. 2007;8:101-12.

[10] Hershko A. Roles of ubiquitin-mediated proteolysis in cell cycle control. Curr Opin Cell Biol. 1997;9:788-99.

[11] Hershko A, Ciechanover A. The ubiquitin system. Ann Rev Biochem. 1998;67:425-79.

[12] Hershko A. The ubiquitin system for protein degradation and some of its roles in the control of the cell division cycle. Cell Death Differ. 2005;12:1191-7.

[13] Kostova Z, Wolf DH. For whom the bell tolls: Protein quality control of the endoplasmic reticulum and the ubiquitinproteasome connection. EMBO J. 2003;22:2309-17.
[14] Melendez A, Talloczy Z, Seaman M, Eskelinen EL, Hall $\mathrm{DH}$, Levine B. Autophagy genes are essential for dauer development and life-span extension in C. elegans. Science. 2003;301:1387-91.

[15] Otto GP, Wu MY, Kazgan N, Anderson OR, Kessin RH. Macroautophagy is required for multicellular development of the social amoeba Dictyostelium discoideum. J Biol Chem. 2003;278:17636-45.

[16] Komatsu M, Kurokawa H, Waguri S, Taguchi K, Kobayashi A, Ichimura Y, et al. The selective autophagy substrate p62 activates the stress responsive transcription factor Nrf2 through inactivation of Keap1. Nat Cell Biol. 2010;12:213-23.

[17] Yu L, Strandberg L, Lenardo MJ. The selectivity of autophagy and its role in cell death and survival. Autophagy. 2008;4:56773.

[18] Onodera J, Ohsumi Y. Ald6p is a preferred target for autophagy in yeast, Saccharomyces cerevisiae. J Biol Chem. 2004;279:16071-6.

[19] Cuervo AM. Chaperone-mediated autophagy: Selectivity pays off. Trends Endocrin Metab. 2010;21:142-50.

[20] Cuervo AM, Stefanis L, Fredenburg R, Lansbury PT, Sulzer D. Impaired degradation of mutant alpha-synuclein by chaperone-mediated autophagy. Science. 2004;305:1292-5.

[21] Kaushik S, Cuervo AM. Chaperone-mediated autophagy. Methods Mol Biol. 2008;445:227-44.

[22] Lamark T, Johansen T. Autophagy: Links with the proteasome. Curr Opin Cell Biol. 2010;22:192-8.

[23] Kalchman MA, Graham RK, Xia G, Koide HB, Hodgson JG, Graham KC, et al. Huntingtin is ubiquitinated and interacts with a specific ubiquitin-conjugating enzyme. J Biol Chem. 1996;271:19385-94.

[24] Wang J, Wang CE, Orr A, Tydlacka S, Li SH, Li XJ. Impaired ubiquitin-proteasome system activity in the synapses of Huntington's disease mice. J Cell Biol. 2008;180:1177-89.

[25] Li X, Wang CE, Huang S, Xu X, Li XJ, Li H, et al. Inhibiting the ubiquitin-proteasome system leads to preferential accumulation of toxic N-terminal mutant huntingtin fragments. Hum Mol Genet. 2010;19:2445-55.

[26] Li XJ, Li S. Proteasomal dysfunction in aging and Huntington disease. Neurobiol Dis. 2011;43:4-8.

[27] Venkatraman P, Wetzel R, Tanaka M, Nukina N, Goldberg AL. Eukaryotic proteasomes cannot digest polyglutamine sequences and release them during degradation of polyglutamine-containing proteins. Mol Cell. 2004;14:95104.

[28] Sieradzan KA, Mechan AO, Jones L, Wanker EE, Nukina N, Mann DM. Huntington's disease intranuclear inclusions contain truncated, ubiquitinated huntingtin protein. Exp Neurol. 1999;156:92-9.

[29] Jana NR, Zemskov EA, Wang G, Nukina N. Altered proteasomal function due to the expression of polyglutamineexpanded truncated $\mathrm{N}$-terminal huntingtin induces apoptosis by caspase activation through mitochondrial cytochrome $\mathrm{c}$ release. Hum Mol Gen. 2001;10:1049-59.

[30] Sapp E, Schwarz C, Chase K, Bhide PG, Young AB, Penney $\mathrm{J}$, et al. Huntingtin localization in brains of normal and Huntington's disease patients. Ann neurol. 1997;42:604-12.

[31] Davies SW, Turmaine M, Cozens BA, DiFiglia M, Sharp AH, Ross CA, et al. Formation of neuronal intranuclear inclusions underlies the neurological dysfunction in mice transgenic for the HD mutation. Cell. 1997;90:537-48.

[32] Kegel KB, Kim M, Sapp E, McIntyre C, Castaño JG, Aronin N, DiFiglia M.Huntingtin expression stimulates endosomallysosomal activity, endosome tubulation, and autophagy. $\mathrm{J}$ Neurosci. 2000;20(19):7268-78. 
[33] Martinez-Vicente M, Talloczy Z, Wong E, Tang G, Koga H, Kaushik S, et al. Cargo recognition failure is responsible for inefficient autophagy in Huntington's disease. Nat Neurosci. 2010;13:567-76.

[34] Cuervo AM. Autophagy and aging-when "all you can eat" is yourself. Sci Aging Knowledge Environ. 2003;2003:pe25.

[35] Bergamini E, Cavallini G, Donati A, Gori Z. The role of macroautophagy in the ageing process, anti-ageing intervention and age-associated diseases. Int J Biochem Cell Biol. 2004;36:2392-404

[36] Zheng S, Clabough EB, Sarkar S, Futter M, Rubinsztein DC, Zeitlin SO. Deletion of the huntingtin polyglutamine stretch enhances neuronal autophagy and longevity in mice. PLoS Genet. 2010;6(2):e1000838.

[37] Metzger S, Saukko M, Van Che H, Tong L, Puder Y, Riess $\mathrm{O}$, Nguyen HP. Age at onset in Huntington's disease is modified by the autophagy pathway: Implication of the V471A polymorphism in Atg7. Hum Genet. 2010;128(4):453-9

[38] Berger Z, Ravikumar B, Menzies FM, Oroz LG, Underwood BR, Pangalos MN, et al. Rapamycin alleviates toxicity of different aggregate-prone proteins. Hum Mol Genet. 2006; $15: 433-42$.

[39] Wu JC, Qi L, Wang Y, Kegel KB, Yoder J, Difiglia M, et al. The regulation of N-terminal Huntingtin (Htt552) accumulation by Beclin1. Acta Pharmacol Sin. 2012;33:743-51

[40] Sarkar S, Davies JE, Huang Z, Tunnacliffe A, Rubinsztein DC Trehalose, a novel mTOR-independent autophagy enhancer, accelerates the clearance of mutant huntingtin and alphasynuclein. J Biol Chem. 2007;282:5641-52.

[41] Ravikumar B, Sarkar S, Rubinsztein DC. Clearance of mutant aggregate-prone proteins by autophagy. Methods Mol Biol. 2008;445:195-211.

[42] Yamamoto A, Cremona ML, Rothman JE. Autophagymediated clearance of huntingtin aggregates triggered by the insulin-signaling pathway. J Cell Biol. 2006;172(5):719-31.

[43] Filimonenko M, Isakson P, Finley KD, Anderson M, Jeong H, Melia TJ, Bartlett BJ, Myers KM, Birkeland HC, Lamark T, Krainc D, Brech A, Stenmark H, Simonsen A, Yamamoto A.The selective macroautophagic degradation of aggregated proteins requires the PI3P-binding protein Alfy. Mol Cell. 2010;38(2):265-79.

[44] Tsunemi T, Ashe TD, Morrison BE, Soriano KR, Au J, Roque RA, Lazarowski ER, Damian VA, Masliah E, La Spada AR. PGC- $1 \alpha$ rescues Huntington's disease proteotoxicity by preventing oxidative stress and promoting TFEB function. Sci Transl Med. 2012;4(142):142ra97

[45] Shin J-Y, Fang Z-H, Yu Z-X, Wang C-E, Li S-H, Li X-J. Expression of mutant huntingtin in glial cells contributes to neuronal excitotoxicity. J Cell Biol. 2005;171:1001-12.

[46] Faideau M KJ, Cormier K, Gilmore R, Welch M, Auregan G, Dufour N, Guillermier M, Brouillet E, Hantraye $\mathrm{P}$, Déglon N, Ferrante RJ, Bonvento G. In vivo expression of polyglutamine-expanded huntingtin by mouse striatal astrocytes impairs glutamate transport: A correlation with Huntington's disease subjects. Hum Mol Genet. 2010;19:3053-7.

[47] Chen LL, Wu JC, Wang LH, Wang J, Qin ZH, Difiglia M, et al. Rapamycin prevents the mutant huntingtin-suppressed GLT-1 expression in cultured astrocytes. Acta Pharmacol Sin. 2012;33:385-92.

[48] Thompson LM, Aiken CT, Kaltenbach LS, Agrawal N, Illes $\mathrm{K}$, Khoshnan A, et al. IKK phosphorylates Huntingtin and targets it for degradation by the proteasome and lysosome. J Cell Biol. 2009;187:1083-99.
[49] Bauer PO, Goswami A, Wong HK, Okuno M, Kurosawa M, Yamada M, et al. Harnessing chaperone-mediated autophagy for the selective degradation of mutant huntingtin protein. Nat Biotechnol. 2010;28:256-63.

[50] Koga H, Martinez-Vicente M, Arias E, Kaushik S, Sulzer D, Cuervo AM. Constitutive upregulation of chaperonemediated autophagy in Huntington's disease. J Neurosci. 2011;31:18492-505

[51] Qi L, Zhang XD, Wu JC, Lin F, Wang J, Difiglia M, et al. The role of chaperone-mediated autophagy in huntingtin degradation. PloS one. 2012;7:e46834.

[52] Cuervo AM, Dice JF. Age-related decline in chaperonemediated autophagy. J Biol Chem. 2000;275:31505-513.

[53] Cuervo AM, Bergamini E, Brunk UT, Dröge W, Ffrench M, Terman A. Autophagy and aging: The importance of maintaining "clean" cells. Autophagy. 2005;1(3):131-40.

[54] Gu X, Greiner ER, Mishra R, Kodali R, Osmand A, Finkbeiner $\mathrm{S}$, et al. Serines 13 and 16 are critical determinants of fulllength human mutant huntingtin induced disease pathogenesis in HD mice. Neuron. 2009;64:828-40.

[55] Steffan JS, Bodai L, Pallos J, Poelman M, McCampbell A, Apostol BL, et al. Histone deacetylase inhibitors arrest polyglutamine-dependent neurodegeneration in Drosophila. Nature. 2001;413:739-43.

[56] Jeong H, Then F, Melia TJ Jr., Mazzulli JR, Cui L, Savas JN, et al. Acetylation targets mutant huntingtin to autophagosomes for degradation. Cell. 2009;137:60-72.

[57] Hockly E, Richon VM, Woodman B, Smith DL, Zhou X, Rosa E, et al. Suberoylanilide hydroxamic acid, a histone deacetylase inhibitor, ameliorates motor deficits in a mouse model of Huntington's disease. Proc Natl Acad Sci USA. 2003;100:2041-6.

[58] Watanabe T, Nagase K, Chosa M, Tobinai K. Schwann cell autophagy induced by SAHA, 17-AAG, or clonazepam can reduce bortezomib-induced peripheral neuropathy. Brit J Cancer. 2010;103:1580-7.

[59] Chen MY, Liao WS, Lu Z, Bornmann WG, Hennessey $\mathrm{V}$, Washington MN, et al. Decitabine and suberoylanilide hydroxamic acid (SAHA) inhibit growth of ovarian cancer cell lines and xenografts while inducing expression of imprinted tumor suppressor genes, apoptosis, G2/M arrest, and autophagy. Cancer. 2011;117:4424-38.

[60] Thomas EA, Coppola G, Desplats PA, Tang B, Soragni E, Burnett R, et al. The HDAC inhibitor $4 \mathrm{~b}$ ameliorates the disease phenotype and transcriptional abnormalities in Huntington's disease transgenic mice. Proc Natl Acad Sci USA. 2008; 105:15564-9.

[61] Jia H, Kast RJ, Steffan JS, Thomas EA. Selective histone deacetylase (HDAC) inhibition imparts beneficial effects in Huntington's disease mice: Implications for the ubiquitinproteasomal and autophagy systems. Hum Mol Genet. 2012;21(24):5280-93

[62] Frank S. Tetrabenazine as anti-chorea therapy in Huntington disease: An open-label continuation study. Huntington Study Group/TETRA-HD Investigators. BMC Neurol. 2009;9:62.

[63] Frank S. Tetrabenazine: The first approved drug for the treatment of chorea in US patients with Huntington disease. Neuropsychiatr Dis Treat. 2010;6:657-65.

[64] Tetrabenazine as antichorea therapy in Huntington disease: A randomized controlled trial. Neurology. 2006;66:366-72.

[65] Sarkar S, Perlstein EO, Imarisio S, Pineau S, Cordenier A, Maglathlin RL, et al. Small molecules enhance autophagy and reduce toxicity in Huntington's disease models. Nat Chem Biol. 2007;3:331-8. 
[66] Williams A, Sarkar S, Cuddon P, Ttofi EK, Saiki S, Siddiqi FH, et al. Novel targets for Huntington's disease in an mTORindependent autophagy pathway. Nat Chem Biol. 2008;4:295 305 .

[67] Balgi AD, Fonseca BD, Donohue E, Tsang TC, Lajoie P, Proud CG, et al. Screen for chemical modulators of autophagy reveals novel therapeutic inhibitors of mTORC1 signaling. PloS One. 2009; 4:e7124.

[68] Tsvetkov AS, Miller J, Arrasate M, Wong JS, Pleiss MA, Finkbeiner S. A small-molecule scaffold induces autophagy in primary neurons and protects against toxicity in a Huntington disease model. Proc Natl Acad Sci USA. 2010;107:16982-7.

[69] Ravikumar B, Vacher C, Berger Z, Davies JE, Luo S, Oroz LG, et al. Inhibition of mTOR induces autophagy and reduces toxicity of polyglutamine expansions in fly and mouse models of Huntington disease. Nat Genet. 2004;36:585-95.

[70] Menzies FM, Huebener J, Renna M, Bonin M, Riess O, Rubinsztein DC. Autophagy induction reduces mutant ataxin3 levels and toxicity in a mouse model of spinocerebellar ataxia type 3. Brain. 2010;133:93-104.

[71] Ravikumar B, Duden R, Rubinsztein DC. Aggregate-prone proteins with polyglutamine and polyalanine expansions are degraded by autophagy. Hum Mol Genet. 2002;11:1107-17.

[72] Crowe JH, Oliver AE, Tablin F. Is there a single biochemical adaptation to anhydrobiosis? Integr Comp Biol. 2002;42:497503.

[73] Colaco C, Kampinga J, Roser B. Amorphous stability and trehalose. Science. 1995;268:788.

[74] Tanaka M, Machida Y, Niu S, Ikeda T, Jana NR, Doi H, et al. Trehalose alleviates polyglutamine-mediated pathology in a mouse model of Huntington disease. Nat Med. 2004;10: 148-54.

[75] Rose C, Menzies FM, Renna M, Acevedo-Arozena A, Corrochano S, Sadiq O, et al. Rilmenidine attenuates toxicity of polyglutamine expansions in a mouse model of Huntington's disease. Hum Mol Genet. 2010;19:2144-53.

[76] Sarkar S, Floto RA, Berger Z, Imarisio S, Cordenier A, Pasco $\mathrm{M}$, et al. Lithium induces autophagy by inhibiting inositol monophosphatase. J Cell Biol. 2005;170:1101-11.

[77] Sarkar S, Krishna G, Imarisio S, Saiki S, O’Kane CJ, Rubinsztein DC. A rational mechanism for combination treatment of Huntington's disease using lithium and rapamycin. Hum Mol Genet. 2008;17:170-8.

[78] Duan W, Guo Z, Jiang H, Ware M, Li XJ, Mattson MP. Dietary restriction normalizes glucose metabolism and BDNF levels, slows disease progression, and increases survival in huntingtin mutant mice. Proc Natl Acad Sci USA. 2003;100:2911-6.

[79] Grumati P, Coletto L, Schiavinato A, Castagnaro S, Bertaggia E, Sandri M, et al. Physical exercise stimulates autophagy in normal skeletal muscles but is detrimental for collagen VIdeficient muscles. Autophagy. 2011;7:1415-23.

[80] Grumati P, Coletto L, Sandri M, Bonaldo P. Autophagy induction rescues muscular dystrophy. Autophagy. 2011;7:426-8.

[81] He C, Sumpter R Jr., Levine B. Exercise induces autophagy in peripheral tissues and in the brain. Autophagy. 2012;8(10):1548-51

[82] He C, Bassik MC, Moresi V, Sun K, Wei Y, Zou Z, et al. Exercise-induced BCL2-regulated autophagy is required for muscle glucose homeostasis. Nature. 2012;481:511-5. 